\title{
Volker Hagedorn: Der Klang von Paris. Eine Reise in die musikali- sche Metropole des 19. Jahrhunderts. Reinbek bei Hamburg: Rowohlt Verlag, 2019. ISBN 3498030353. 416 Seiten.
}

Seinem 1982 im Passagen-Werk auf Deutsch und Französisch erstveröffentlichten Exposé „Paris, die Hauptstadt des XIX. Jahrhunderts“ stellt Walter Benjamin ein Motto voran, das ihn möglicherweise zu seinem Titel inspirierte. Es handelt sich dabei um ein Zitat aus dem 1897 ebenfalls zweisprachig (vietnamesisch-französisch) in Hanoi publizierten Gedichtband Paris, capitale de la France von Nguyên-Trong-Hiêp, der 36, jeweils mit einem erklärenden ,Kommentar ' versehene, poetische Skizzen oder Reiseimpressionen versammelt und, der kolonialistischen Ideologie das Wort redend, ein überwältigend positives Bild der Modernität der französischen Hauptstadt zeichnet. Diesem poetisch-didaktisch verbrämten Echo eines mit einer Delegation in das Paris des späten 19. Jahrhunderts reisenden Beamten und „Commandeur de la Légion d'honneur“ steht der distanzierte Blick des Philosophen Benjamin gegenüber, der ab den späten 1920er Jahren die visionäre Modernität der französischen Metropole des 19. Jahrhunderts zu fassen sucht. In seinem Exposé ortet Benjamin diese in den Stahlkonstruktionen der Passagen, Ausstellungshallen und Bahnhöfe, den Panoramen, der Erfindung der Fotografie, den Weltausstellungen, den Interieurs und privaten Sammlungen, dem Phänomen des urbanen Flaneurs und den aus dem Haussmannschen Urbanismus entstandenen Straßenfluchten. In dem von Benjamin skizzierten Stadtraum des aus der industriellen Revolution hervorgegangenen Finanzkapitals ist Heinrich Heines Paris als „Sammelplatz [...] geistiger Notabilitäten“" und sich gegenseitig befruchtender Möglichkeiten zwar eingeschrieben, allerdings nicht als lebendige Bewegung, sondern als „Stillstand“ und „Traumbild“ (Benjamin 1982, 55). Was Walter Benjamin zur Weltausstellung des Jahres 1867 und der dort zelebrierten Warenästhetik schreibt, nämlich

[d]ie Phantasmagorie der kapitalistischen Kultur erreicht auf der Weltausstellung von 1867 ihre strahlendste Entfaltung. Das Kaiserreich steht auf der Höhe seiner Macht. Paris bestätigt sich als Kapitale des Luxus und der Moden. Offenbach schreibt dem pariser [sic] Leben den Rhythmus vor. Die Operette ist die ironische Utopie einer dauernden Herrschaft des Kapitals[,] (Benjamin 1982, 51-52)

scheint auch für das von Nguyên-Trong-Hiên beschriebene Paris der Troisième République zu gelten, wenn letzterer wie folgt zur 1875 eröffneten Opéra Garnier ,dichtet': 
XX

Sur le grand boulevard s'élève un magnifique théâtre.

Dans l'intérieur, la dordure [sic] et la peinture brillent d'un vif éclat sous l'influence des milliers de lumières.

On entend des artistes qui chantent très bien, et surtout de fort jolies danseuses.

Semblable aux fées qui jouent la pièce de Nghè-thuong

Dans le Palais de Quan-han.

\begin{abstract}
Le Grand-Opéra est un bâtiment très beau et très vaste, il est entièrement construit en belles pierres de taille. La construction devait durer plusieurs années. C'est le premier théâtre de Paris. Sa hauteur égale à celle des maisons de sept étages ; ses décorations intérieures, peinture et dorure, sont superbes et brillent d'un éclat merveilleux. Sa beauté peut rivaliser avec celle des palais des rois. Son Excellence M. le Président de la République avait invité la mission à assister à la représentation, nous avons pu voir des artistes charmants qui chantent très bien et surtout de fort jolies jeunes filles qui dansent d'une manière fort gracieuse. (Nguyên-Trong-Hiên 1897, o.S.)
\end{abstract}

Auf der "Reise in die musikalische Metropole des 19. Jahrhunderts“, zu der Volker Hagedorn in seinem hier besprochenen Band Der Klang von Paris einlädt, spielt die Opéra Garnier allerdings noch keine Rolle. Sie beginnt 1821, in Napoleon Bonapartes Todes- und Gustave Flauberts Geburtsjahr, als der junge Hector Berlioz für seine Reise mit der Postkutsche zum Medizinstudium nach Paris aus dem heutigen Département Isère in die Hauptstadt nahezu fünf ganze Tage braucht (13), und endet 1867, im Jahr der zweiten Pariser Weltausstellung, als „Paris [...] nach London die größte Stadt der Welt [ist], mit jetzt mehr als dreimal so vielen Einwohnern wie Berlin" (288), und über acht Bahnhöfe verfügt. Zwischen dem 1. April und dem 30. November 1867 strömen Menschen aus Europa und der ganzen Welt in den Ausstellungspalast auf dem Champ de Mars - in der einschlägigen Literatur ist von 11 bis 15 Millionen Besuchern die Rede (cf. Ageorges 2006) -, um dort u. a. „eine alles in allem 50 Tonnen schwere Kanone der Firma Friedrich Krupp" zu bestaunen, die mit einem Sonderzug von Essen nach Paris transportiert wurde (339). Es handelt sich vielleicht um einen Gemeinplatz, wenn Hagedorn, um die technischen, gesellschaftlichen und künstlerischen Umbrüche zwischen 1821 und 1867 zu illustrieren, immer wieder die das Raum- und Zeitempfinden revolutionierenden neuen Transportmittel ins Spiel bringt. Aber gibt es ein treffenderes Beispiel, um die „Zeit beschleunigter Fortschritte in Wissenschaft und Technik" (21) auf den Punkt zu bringen, als Frédéric Chopin im November 1846 auf seinem Weg von Nohant nach Paris zu begleiten (169-171), mit ihm über die 
Geschwindigkeit zu staunen und gleichzeitig zu erfahren, dass bereits ein halbes Jahr später „die französischen Eisenbahnaktien um bis zu 75\% abstürzen“ würden (172)? „Tausende von Franzosen werden ihre Arbeit verlieren, und diese Krise bereitet, wie die Missernten, einer weiteren Revolution das Feld“ (172).

Hagedorns Reise in die Pariser Musikwelt von der Restauration bis kurz vor dem Deutsch-Französischen Krieg von 1870/71 entspinnt sich also nicht allein entlang der Errungenschaften der industriellen Revolution, sondern nimmt auch ihre Schattenseiten in den Blick, erörtert Krisen und Revolutionen, demographische wie politische Entwicklungen, die Verbürgerlichung der Gesellschaft auf der einen, die Verelendung der Massen auf der anderen Seite. Sie orientiert sich an großen literarischen und künstlerischen Figuren der Epoche (u. a. Heinrich Heine, Victor Hugo, Stendhal, Honoré de Balzac, George Sand, Gustave Flaubert, Jules und Edmond de Goncourt, Charles Baudelaire, Eugène Delacroix, die Impressionisten), blickt auf die schlechten hygienischen Bedingungen in der Stadt und die daraus resultierenden Epidemien (Choleraepidemie von 1832 und 1849, 89-93 und 211), sondiert die Neugestaltung der capitale unter Baron Haussmann (inklusive Enteignungen und Immobilienspekulationen, 254) und kommentiert nebst anderem etwa mit Nadar alias Gaspard-Félix Tournachon eine der zukunftsweisendsten Erfindungen in der Zeit der Juli-Monarchie, die Fotografie:

Tatsächlich entquellen diesem Jahrhundert die Ideen in solcher Überfülle, in so glorreicher Hast, dass sie gar keiner Inkubationszeit zu bedürfen scheinen. Der Gedanke jagt der Tat zu. Kaum hat der Dampf die Entfernung verkürzt, wird er durch die Elektrizität verdrängt. [...] Doch müssen nicht all diese neuen Wunder vor dem allerunbegreiflichsten verblassen - dem Wunder, das endlich auch dem Menschen Schöpfermacht zu verleihen scheint, wenn er das körperlose Phantom materialisiert, das, kaum erblickt, verschwindet, ohne einen Hauch auf dem Kristall des Spiegels, ohne ein Zittern auf der Oberfläche des Wassers zurückzulassen? (246-247)

Hagedorns Projekt, „Paris [...] als Zentrum europäischer Musik im 19. Jahrhundert“ zu erkunden (Klappentext), versteht sich - das dürfte nun klar geworden sein - als breit angelegte kulturgeschichtliche Unternehmung mit einem Schwerpunkt auf dem musikalischen Geschehen, wobei die einfühlende Imaginationskraft des im frühen 21. Jahrhundert lebenden Autors bei der Rekonstruktion der Gedanken- und Erfahrungswelt der von ihm skizzierten historischen Persönlichkeiten mitunter literarische Dimensionen annimmt. So begleiten die Leser etwa Richard Wagner zu Giacomo Meyerbeer und sehen zu, wie letzterer die Zeitung zusammenfaltet (123-124), beobachten Chopin beim Klavierunterricht (181-184) oder belauschen Gespräche zwischen Chopin und Delacroix (213) oder Pauline Viardot und Hector Berlioz (280-282), um hier nur Beispiele zu nennen. In einem etwas ungewöhnlich angelegten Verweisapparat („Anmerkungen“, 351-381) dokumentiert der Autor zwar sehr akribisch die Quellen, die er für die Rekonstruktion der oben genannten und ähnlicher Szenen heranzieht (Briefe, Tagebü- 
cher, Memoiren, Autobiographien, Presseartikel, Reportagen u. Ä.), lässt dabei aber jede kritische Auseinandersetzung mit eben denselben Quellen vermissen. Dies schmälert nun nicht das Lesevergnügen, wirft aber die Frage nach der Glaubwürdigkeit und der dokumentarischen Seriosität der so packenden ,Erzählung' auf, ja vielleicht sogar jene nach der Gattung, mit der man es hier zu tun hat.

In jedem Fall scheint Hagedorn mit Strategien des Literarischen zu liebäugeln, was zu allererst in den Kapitelüberschriften zum Ausdruck kommt. Den sechs Kapiteln des chronologisch angelegten Bandes sind nebst Überschriften auch Teaser vorangestellt, die in aller Kürze auf inhaltliche Details verweisen und an Voltaires Verfahren in den contes philosophiques erinnern. ${ }^{2}$ So verlautet etwa der Teaser zu Kapitel 4 „Chopin, Barrikaden und ein Prophet (1846-1849)“: „Chopin verliert George Sand, flieht vor der Revolution und fährt mit Delacroix spazieren. Meyerbeer lässt einen marxistischen Propheten in der Oper elektrisch beleuchten" (165; die Teaser zu den einzelnen Kapiteln sind übrigens auch im Inhaltsverzeichnis angeführt). Eine weitere „literarische“ Besonderheit des Bandes besteht in der Verknüpfung der ,Reise in die Vergangenheit mit insgesamt 12 Einschüben, in denen Hagedorn den Leser sozusagen in die Gegenwart zurückholt, ihn an seinen „Spurensuchen“ (30) teilhaben lässt, ihn zum ,Lokalaugenschein' an Originalschauplätze in Paris oder zu Treffen mit Expert*innen wie dem Pleyel-Kenner Olivier Fadini (173-179), der Kultursopranistin Sabine Devieilhe (153-155) oder zu Michel Austin und Monir Tayeb - die beiden verwalten ein digitales Berlioz-Archiv und betreiben die Webseite hberlioz.com nach Edinburgh mitnimmt (298-302). Diese Einschübe thematisieren den Autor als Person und Suchenden, als Beobachtenden und Wahrnehmenden, als Menschen seiner Zeit, der die Terroranschläge im November 2015 ebenso im Auge hat (154) wie die politischen und wirtschaftlichen Entwicklungen unter Emmanuel Macron (99) oder im RER in Richtung Roissy einem Paar - „ [...] vielleicht Geschwister, vielleicht aus dem Libanon, Mitte zwanzig, er mit dichtem Bart, sie mit langem Zopf, vor den Türen einander gegenüber, halb tanzend, gesprochener Text, gesungener Refrain, harter Beat aus der Box, Leben" (347) - beim Rappen zuhört. Insgesamt hält Hagedorns ,allwissender Erzähler' mit seinen Einschätzungen nicht hinter dem Berg und lässt an jenen in Balzacs Romanen denken, wenn in Der Klang von Paris z. B. zu lesen ist: „Es ist nicht fair, wie Berlioz in seinen Memoiren den Chef des Conservatoire inszeniert [...]" (28).

Die Beschreibungen der aufeinander folgenden Musikergenerationen (von der Romantik als Modebewegung bis hin zu Offenbachs Operetten gegen Ende des Empires und der vom Kaiser angeordneten Pariser Erstaufführung von Wagners romantischer Oper Tannhäuser $^{3}$, der Abfolge verschiedener Stilrichtungen und Aufführungspraktiken, der persönlichen Schicksale der Musiker, Diven und Theatermacher, der Rivalitäten, Auf- und Abstiege - dies alles stets verwoben mit den bereits angesprochenen politischen, ökonomischen gesellschaftlichen und kulturellen Entwicklungen -, sind von einer nur schwer zu resümierenden Dichte. Hervorgehoben werden sollen hier nur die immer wieder fesselnden Skizzen diverser Aufführungen und Konzerte, die einen faszinierenden Einblick in die sich stetig entwickelnde und verändernde Musik- und Theaterpraxis zwischen 1821 und 1867 
geben, darunter die Uraufführungen von Gioachino Rossinis Guillaume Tell (51-54) und der Symphonie fantastique von Berlioz, die den 19-jährigen Franz Liszt begeistert (67-69); eines der legendären Konzerte von Niccolò Paganini im Jahr 1831, den George Sand in bester physiognomischer Tradition in einem Artikel für den Figaro beschreibt (74); die Uraufführung von Giacomo Meyerbeers Robert le diable (1849), wegen der eine Abstimmung in der Nationalversammlung entfallen musste (217-223); die ersten großen Erfolge des Direktors der Bouffes-Parisiens Jacques Offenbach mit Orphée aux Enfers (264-272) und der musikalischen Politsatire Sultan Barkouf, einem Auftragswerks für die Opéra-Comique, für das Eugène Scribe das Libretto verfasste (303-307); oder die schwierige Erstaufführung des Tannhäuser (310-316), welche die Diplomatengattin Pauline von Metternich wie folgt kommentiert: „In fünfundzwanzig Jahren wird ganz Paris Wagner applaudieren.“ (315)

In seinen Skizzen berücksichtigt Hagedorn übrigens wiederholt nicht nur die allgemeine Misogynie in der Musikwelt - im Besonderen verweist er auf die schwierige Situation von Komponistinnen wie Louise Bertin, die dem abwertenden Frauenbild zu trotzen suchen (71-72), oder die Tatsache, dass Frauen bei der Uraufführung des Tannhäuser der Zugang zum Parkett verwehrt wurde (311) -, sondern zeichnet durch den ganzen Band hindurch auch die Spuren des zunächst latenten und ab der Mitte des 19. Jahrhunderts immer offensichtlicheren Antisemitismus nach (z. B. 125-126).

Bei der Lektüre von Der Klang von Paris wird nicht zuletzt eines klar: nämlich wie stark Philosophen wie Walter Benjamin das Bild von Paris im 19. Jahrhundert und den intellektuellen Blick auf bestimmte historische Figuren geprägt haben. Dieser Blick, diese Meinung, muss heute differenzierter ausfallen. „Offenbach schreibt dem pariser [sic] Leben den Rhythmus vor. Die Operette ist die ironische Utopie einer dauernden Herrschaft des Kapitals“, verlautet Walter Benjamin. Hagedorn erkennt in der Opéra-Bouffe des Jacques Offenbach mehr, anderes: „Das Zerbrechen der großen Erzählung spiegelt sich bei Offenbach, die Entzauberung der Welt in einer Zeit der Beschleunigung, ihre ,transformation', über deren Baustellen man in Paris täglich stolpert. Er wird als erster einen Bahnhof auf die Bühne bringen und eine Reise zum Mond..." (272) Allein auf Grund solcher Beobachtung, aber nicht nur, ist Hagedorns Der Klang von Paris sozusagen eine Reise wert.

\section{Doris EIBL (Innsbruck)}

\section{Endnoten}

1 „Paris ist nicht bloß die Hauptstadt von Frankreich, sondern der ganzen zivilisierten Welt, und ist ein Sammelplatz ihrer geistigen Nobilitäten. Versammelt ist hier alles, was groß ist durch Liebe oder Haß, durch Fühlen oder Denken, durch Wissen oder Können, durch Glück oder Unglück, durch Zukunft oder Vergangenheit. Betrachtet man den Verein von berühmten oder ausgezeichneten Männern, die hier zusammentreffen, so hält man Paris für ein Pantheon der 
Lebenden. Eine neue Kunst, eine neue Religion, ein neues Leben wird hier geschaffen, und lustig tummeln sich hier die Schöpfer einer neuen Welt. Die Gewalthaber gebärden sich kleinlich, aber das Volk ist groß und fühlt seine schauerlich erhabene Bestimmung." (10. Februar 1832) (Heine 1961, 413-414)

2 Z. B. in Candide ou l'Optimisme: "Chapitre VI. Comment on fit un bel auto-da-fé pour empêcher les tremblements de terre, et comment Candide fut fessé” (Voltaire 1877, 148).

3 Wagners Musik wurde in Paris als „grauenhafte Zukunftsmusik“, als „musique de l'avenir“ empfunden (290).

\section{Bibliographie}

Ageorges, Sylvain: Sur les traces des expositions universelles, Paris, 1855-1937. Paris: Éditions Parigramme, 2006, http://www.expositions-universelles.fr/1867-exposition\%20universelle-paris. html (Zugriff 19.12.2019).

Benjamin, Walter: „Paris, die Hauptstadt des XIX. Jahrhunderts“. In: Benjamin, Walter: Das Passagen-Werk. Bd. 1. Frankfurt a. M.: Suhrkamp, 1982, 45-59.

Heine, Heinrich: „Französische Zustände - Artikel III“. In: Heine, Heinrich: Werke und Briefe. Bd. 4. Berlin: Aufbau, 1961, 403-414.

Nguyên-Trong-Hiên: Paris, capitale de la France. Recueil de vers. Hanoï: Imprimerie typo-lithographique F.-H. Schneider, 1897, https://gallica.bnf.fr/ark:/12148/bpt6k58158290/f9.image. texteImage (Zugriff 19.12.2019).

Voltaire: „Candide, ou l'Optimisme“. In: Voltaire: CEuvres complètes de Voltaire. Vol. 21. Paris: Garnier, 1877, 137-218. 